\title{
THE TULLY-FISHER RELATION USING THE CO-LINE FOR INTERACTING GALAXIES
}

\author{
Y. TUTUI AND Y. SOFUE \\ Institute of Astronomy, University of Tokyo \\ Mitaka, Tokyo 181, Japan
}

The CO Tully-Fisher (TF) relation is able to measure the distances to farther galaxies than the HI TF relation reaches (Sofue et al. 1997). The galaxies observed for the $\mathrm{CO} \mathrm{TF}$ relation at the high redshift are often infrared luminous galaxies. The fraction of interacting galaxies is large in the infrared luminous galaxies. Therefore, first we have analyzed the CO and HI linewidths for nearby interacting galaxies in order to examine the influence of the interaction on the linewidths. We found that HI linewidths for the interacting galaxies are significantly larger than $\mathrm{CO}$ (Tutui and Sofue 1997a). It suggests that the influence of the interaction is smaller at the inner region of galaxies where the $\mathrm{CO}$ gas exists, and that the $\mathrm{CO} \mathrm{TF}$ relation is more reliable tool for the galaxies which have the evidence of the galaxy interaction as well as galaxies in rich clusters.

Secondly, in order to analyze the consistency of the $\mathrm{CO}$ and HI linewidths, we have analyzed the relation between the $\mathrm{CO}$ and $\mathrm{HI}$ linewidths for isolated field galaxies including the Cepheid calibrators (Tutui and Sofue, 1997b). We found that the $\mathrm{CO}$ linewidths are larger than $\mathrm{HI}$ for giant galaxies, and the $\mathrm{HI}$ linewidths are larger than $\mathrm{CO}$ for dwarf galaxies. Based on this correlation, the coefficients of the $\mathrm{CO} \mathrm{TF}$ relation should be changed from those of the HI TF relation. The differences in the coefficients can be explained by the synthetic rotation curve model proposed by Persic et al.(1996) and the different gas distribution in galaxies.

\section{References}

Persic, M., Salucci, P., Stel, F., 1996, MNRAS 281, 27

Sofue. Y., Tutui, Y., Honma, M., Ichikawa, T., Wakamatsu, K., Kazes, I. \& Dickey, J. 1997 , in these proceedings

Tutui Y., Sofue Y. 1997a, submitted to PASJ

Tutui Y., Sofue Y. 1997b, A\&A in press 